Universidad de Lima

Facultad de Psicología

Carrera de Psicología

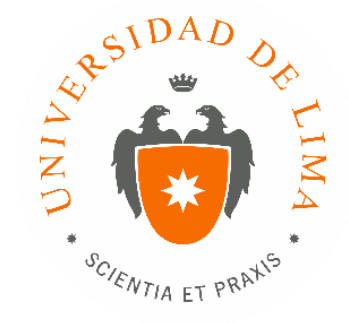

\title{
EXPERIENCIA EN EL ÁREA DE NEUROPSICOLOGÍA DE UN HOSPITAL
}

Trabajo de suficiencia profesional para optar el título profesional de Licenciado en Psicología

\author{
Juan Manuel Velarde Ballivian
}

Código 20112451

$$
\text { Lima - Perú }
$$

Febrero de 2019 




\section{EXPERIENCIA EN EL ÁREA DE NEUROPSICOLOGÍA DEL HOSPITAL CENTRAL FAP}




\section{TABLA DE CONTENIDO}

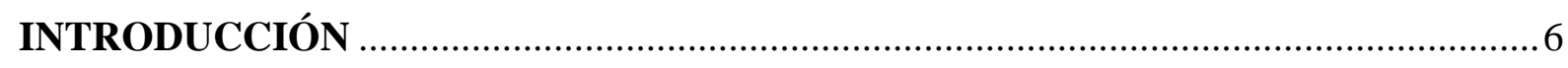

CAPÍTULO I: IDENTIFICACIÓN DEL PROBLEMA ...............................................

CAPÍTULO II: DESCRIPCIÓN DE LAS ACTIVIDADES REALIZADAS ................. 9

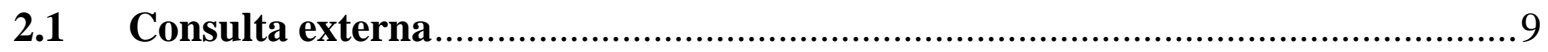

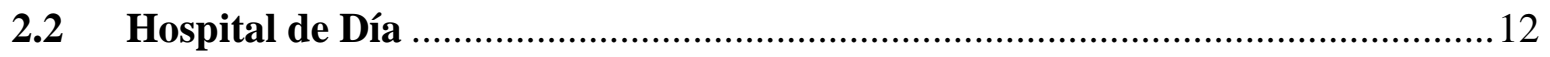

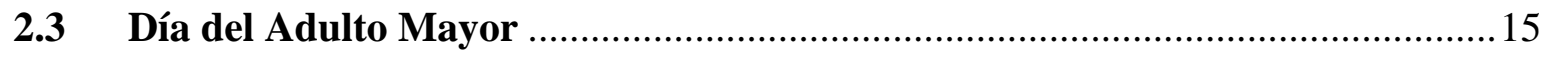

CAPÍTULO III: RESULTADOS DE LA INTERVENCIÓN...................................... 17

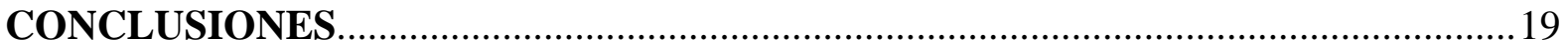

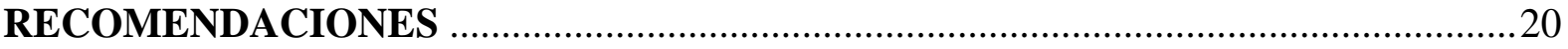

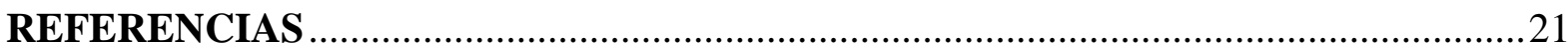

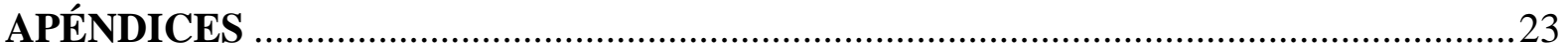




\section{ÍNDICE DE APÉNDICES}

APÉNDICE 1: TEST DE APRENDIZAJE AUDITIVO-VERBAL DE REY .................24

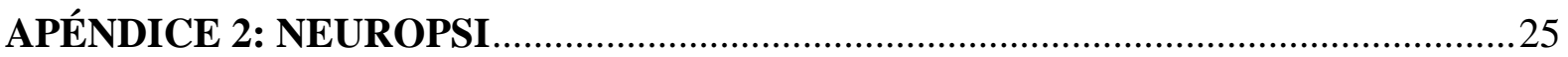

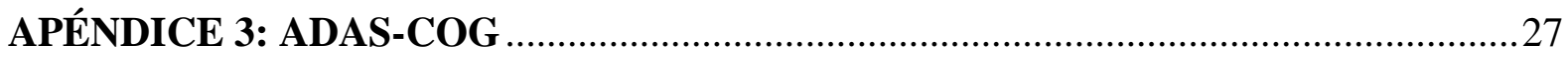

APÉNDICE 4: TEST DE ALTERACIÓN DE LA MEMORIA ........................................ 31 


\section{INTRODUCCIÓN}

En el presente trabajo se describirá, de manera general, el problema que presentan los adultos mayores respecto del deterioro cognitivo, para luego centrar el tema en la coyuntura nacional del Perú y la urgente necesidad de contar con especialistas que puedan velar por el cuidado y la integridad de estos pacientes, para así otorgarles la ayuda pertinente y brindarles una mejor calidad de vida. Asimismo, se revisará la respuesta del Hospital Central de la Fuerza Aérea del Perú (FAP) para combatir esta problemática, con la creación de un departamento de Geriatría que está conformado por un grupo de multidisciplinario de especialistas en el adulto mayor. El presente documento muestra la experiencia del autor como interno de Psicología, durante el tiempo que estuvo rotando en el área de Neuropsicología, realizando entrevistas, evaluaciones y diagnósticos a los pacientes adultos mayores bajo la supervisión del doctor Igor Infantes Portilla, jefe y fundador del área. Se describirán las funciones como interno y las diversas actividades que realizaba en los distintos niveles de atención, que eran, consulta externa y Hospital de Día. Posteriormente, se expondrán los resultados cuantitativos y cualitativos obtenidos relacionados con la atención a los pacientes y las actividades recreativas que se realizaban dentro del área de Neuropsicología del Hospital Central FAP. Luego de esto, se procederá a brindar las conclusiones a las que se ha llegado luego de cuatro meses de práctica en el área, para así poder brindar recomendaciones que favorecerían las condiciones de atención a la población FAP adulta mayor en condición de retirados y a sus familiares directos que se atienden en el hospital. 


\section{CAPÍTULO I: IDENTIFICACIÓN DEL PROBLEMA}

El deterioro cognitivo es un problema serio que se va acentuando con el paso de los años. La Organización Mundial de la Salud (OMS, 2016) señala que, aproximadamente, un 15\% de los adultos mayores padecen de algún trastorno mental; por ejemplo, la enfermedad de Alzheimer, donde se aprecia un deterioro a nivel cognitivo.

Envejecer es parte del ciclo de la vida; es decir, todos los seres humanos pasan por esa etapa y por ello es un tema de suma importancia, y más ahora que la tasa de natalidad a nivel mundial está disminuyendo y el promedio de vida está aumentando. Se espera que haya un aumento de 1.348,3 millones de adulos mayores para el 2030.

Existen enfermedades que en sus inicios tempranos pueden confundirse con un deterioro cognitivo esperado para la edad y debido a ello es que hay personas que no reciben el tratamiento necesario, como en el caso de la enfermedad de Alzheimer, que ocasiona un daño irreversible en el sujeto, destruye lentamente su memoria y otros procesos cognitivos hasta que éste es incapaz de actuar por su propia cuenta (National Institute of Aging, 2015). Es por esto que una detección rápida podría marcar una diferencia significativa en la vida de la persona afectada.

De acuerdo al Instituto Nacional de Estadística e Informática (2015) la tasa de la población adulta mayor en el Perú asciende a 3.011.050 personas; esto representa un 9,7\% de la población que es propensa a presentar enfermedades mentales que perjudican sus procesos cognitivos. Las personas entre 70 y 79 años tienen un aumento en el $10 \%$ en la prevalencia del deterioro cognitivo (Custodio et al., 2013).

Un gran número de adultos mayores en nuestro país no cuentan con las condiciones óptimas para disfrutar su vejez, esto se puede deber a que las familias no saben cómo tratarlos, o porque cubrir todas sus necesidades es un gasto muy grande. De acuerdo al Instituto Nacional de 
Estadística e Informática (2018), el 43,1\% de los adultos mayores no pertenece a la Población Económicamente Activa (PEA), por lo que se convierten en una carga para su entorno. Esto puede tener relacion con que el 5\% de las personas mayores de 65 años presentan limitaciones funcionales en las actividades de la vida diaria (Chumpitaz y Moreno, 2016).

En el país, si bien la salud mental cada vez gana mayor importancia, aún no se pueden cubrir todas las necesidades de la población debido al reducido número de psiquiatras y psicólogos clínicos. Dentro de la psicología clínica existe la rama llamada neuropsicología que es donde convergen la psicología y la neurología. Esta se encarga del diagnóstico y tratamiento de los problemas mentales (cognitivos, emocionales y conductuales) que afectan el correcto funcionamiento del cerebro. Esta rama es la principal encargada de velar por la salud mental de los adultos mayores, pero lamentablemente, como se mencionó anteriormente, hay una gran carencia en este campo. Esto es, porque no hay suficientes centros especializados en neuropsicología para tratar al gran número de adultos mayores del país.

En relación a ello, el Hospital Central de la Fuerza Aérea del Perú (FAP), que brinda servicios al personal de dicha arma en actividad y retirados, al igual que a sus familiares, se vio en la necesidad de cubrir dicha carencia. Es por este motivo que el doctor Igor Infantes Portilla, neuropsicólogo clínico, abre el área de neuropsicología dentro del departamento de Geriatría en este hospital, para así poder atender a los adultos mayores que presentan distintos tipos de trastornos relacionados con las funciones superiores y las estructuras mentales. 


\section{CAPÍTULO II: DESCRIPCIÓN DE LAS ACTIVIDADES Y TAREAS REALIZADAS}

Durante el internado del autor del presente informe en el Hospital Central FAP, rotó por diversas áreas en la sección Psicología, cada área contaba con una o dos psicólogas encargadas. Las rotaciones duraban dos meses y las áreas por las que rotó fueron Psiquiatría, Familia, Pediatría, Endocrinología, Adolescentes, Aeronáutica, Psicología Geriátrica y Neuropsicología. Fue solo en Neuropsicología donde rotó 4 meses ya que ingresó al hospital meses antes de iniciar su internado bajo la figura de practicante y se dio la posibilidad de repetir un área de su elección. A continuación, se explicarán las actividades y tareas realizadas en el área de Neuropsicología.

\subsection{Consulta externa}

Durante la rotación del autor del presente informe en Neuropsicología realizó diversas actividades, siendo la consulta externa la más importante. Esta constaba de visitas diarias de adultos mayores derivados por geriatras y neurólogos, principalmente, para ser evaluados. El objetivo de esta consulta es hacer una evaluación completa al adulto mayor acerca de los niveles en los que se encuentran sus procesos cognitivos.

Los adultos mayores no pueden simplemente sacar una cita y acudir a consulta. Ellos deben ser derivados por otros especialistas para cerciorarse que sus dificultades cognitivas no son causadas por una condición médica preexistente como la diabetes, infección urinaria, infartos vasculares, entre otros. En ocasiones esto causaba malestar en los pacientes que llegaban directamente para una revisión y no podían ser atendidos, pues solo se atienden los pacientes derivados de otras especialidades. 
Este proceso podía demorar entre uno a dos meses, lo que dificultaba seriamente la atención a la población FAP que necesitaba ser evaluada.

La consulta externa con el doctor Igor Infantes constaba de cuatro citas, él recomendaba dos por semana para acabar lo antes posible. En la primera cita era necesario que el adulto mayor viniera acompañado con una persona cercana a él, podía ser un familiar, su cuidador o alguien con quien conviva, para corroborar la información que se solicitaría. Esto en ocasiones genera malestar en los pacientes porque las enfermeras que agendan las citas no les informaban que debían ir acompañados. Las siguientes tres sesiones eran principalmente aplicación de pruebas, una cada día, terminando el último día con una derivación con el geriatra para que este pueda visualizar los resultados de las evaluaciones que se adjuntaban a la historia clínica del paciente.

En la primera consulta se le explicaba al paciente que la evaluación no se iba a poder realizar en una sola sesión. Esto resulta una sorpresa para varios de los adultos mayores. Luego se inicia preguntando acerca de su estado anímico, si salía a la calle y con qué frecuencia, esto porque la depresión puede alterar los procesos cognitivos. Además, se ahondaba acerca de los olvidos que tenía el adulto mayor, estos podían ser acerca del nombre de las cosas o personas, lugares, actividades que realizaba, sucesos de su vida, reconocimiento de las personas de su entorno, entre otros, lo que demostraba el tipo de dificultades en la memoria que podía tener. Asimismo, se hacían preguntas para saber si el paciente estaba orientado en tiempo, espacio y lugar. Tanto el doctor Infantes como el practicante podía realizar la entrevista, si bien él era el que la dirigía el autor del presente informe participaba en todo momento. Inclusive el doctor solía hacer preguntas al practicante durante la 
entrevista, con el fin de que pudiera conectar la información y empezar a pensar en posibles diagnósticos.

La segunda sesión se centraba en la evaluación del Test de Aprendizaje AuditivoVerbal de Rey (Infantes, 2016). En esta prueba se le lee al paciente 15 palabras y se mide cuántos intentos le toma al evaluado poder repetirlas todas. Esto se va registrando en la hoja y al finalizar la prueba se ve una curva de aprendizaje que puede tener diversas formas y es muy útil para ver la evolución en el tiempo, ya que se puede comparar la curva actual con la que se tomó anteriormente en otra consulta. El doctor Infantes y el practicante se turnaban en las evaluaciones las cuales, por lo general, eran supervisadas por aquel salvo cuando ocurrían errores en el sistema de citas y había dos pacientes asignados a la misma hora; en esos momentos, el practicante se retiraba a otro ambiente para hacer la evaluación.

En la tercera sesión se evaluaba otra prueba neuropsicológica, el Neuropsi (Infantes, 2016). Esta prueba es muy completa ya que mide distintos procesos cognitivos como orientación en tiempo, espacio y persona, lo que indica si el adulto mayor sabe dónde está, en qué momento del día y quién es; mide su atención con ejercicios de repetición de dígitos de manera inversa; evalúa la codificación diciéndole al adulto mayor una lista de palabras y este tiene que repetirlas, dándose hasta tres oportunidades si es que el adulto mayor no logra repetirlas correctamente con la variación de que cada vez que las palabras son leídas están en un orden distinto. También mide el lenguaje, mediante una serie de láminas que se le presentan al adulto mayor quien tiene que verbalizar el nombre del objeto, animal o parte del cuerpo que está viendo.

La prueba Neuropsi también mide comprensión lectora: el paciente debe leer una pequeña historia y luego responder una serie de preguntas sobre lo que entendió; 
posteriormente, se evalúan las funciones ejecutivas a nivel conceptual (semejanzas y cálculo) y motora, así como las funciones de evocación haciéndole repetir las palabras que se le dijeron cuando se evaluaba codificación. Esta prueba es extensa por lo que a los adultos mayores les tomaba aproximadamente una hora resolverla y, en algunos casos, era necesario hacerla en dos sesiones.

En la última sesión, podían ocurrir dos escenarios: si el diagnóstico ya estaba decidido solo se realizaba la devolución de los resultados; en caso aún quedara alguna duda, se evaluaba una tercera prueba, el Adas-Cog (Infantes, 2011) que mide once aspectos distintos: inteligibilidad, comprensión, falta de palabras, recuerdo de palabas, denominación, orientación, orientación de órdenes, praxias, praxias constructivas, reconocimiento de palabras y recuerdo de palabras.

En todo momento de las evaluaciones el practicante se encontraba presente tomando apuntes o aplicando las distintas pruebas junto al doctor Infantes. Usualmente, después de cada consulta, el doctor y practicante se quedaban unos minutos en el consultorio discutiendo el caso para así llegar al diagnóstico. Además, solían hacer comparaciones y distinciones entre diversos diagnósticos de los pacientes a modo de aprendizaje y entrenamiento.

\subsection{Hospital de Día}

Dentro del departamento de Geriatría, una de las actividades más importantes es el Hospital de Día, que es un programa interdisciplinario donde distintos especialistas evalúan a adultos mayores en una sola mañana, para hacerles un chequeo general y así poder hacerles seguimiento para ver cómo van evolucionado y si se están adhiriendo o no a los tratamientos asignados. 
Dentro de este programa trabajan el área de Neuropsicología haciendo diversas evaluaciones de deterioro cognitivo, entrevistas al adulto mayor, al igual que a la familia y al cuidador en caso haya uno, evaluaciones acerca de su estado anímico para revisar si tiene depresión, ansiedad u otros problemas emocionales.

Asimismo, el adulto mayor es revisado por las áreas de Odontología, Medicina Geriátrica, Nutrición, Terapia Ocupacional, Terapia Física, Asistenta Social y, finalmente, las enfermeras que son las encargadas de capacitar a los cuidadores acerca de cómo cubrir las necesidades de estos.

Una vez que el adulto mayor ha pasado por todas las áreas, los especialistas se reúnen y uno a uno va exponiendo lo encontrado; de este modo, todos tienen una idea general de cómo le estaba yendo y si necesitaba apoyo en algún área. Mientras que cada uno comentaba lo que concluyeron de la cita con el paciente, la enfermera anotaba los puntos más relevantes para hacer un resumen de las problemáticas encontradas.

Luego de exponer los resultados se inicia un debate sobre cómo proceder con el paciente porque según los resultados de las evaluaciones realizadas puede decidirse darle de alta, que continúe en el programa, o retirarlo de este. Se puede retirar a un adulto mayor porque este no sigue las indicaciones dadas y como está desaprovechando la oportunidad que se le está dando, otro adulto mayor podría tomar su lugar. Del mismo modo, se discute sobre cuándo va a ser su próxima consulta por Hospital de Día.

Los adultos mayores que ingresan a este programa son personas que por alguna razón se les considera en riesgo, por caídas reiteradas, fallecimiento de la pareja, diversas enfermedades físicas, por un declive en los procesos cognitivos, por falta de cuidado por parte de la familia, entre otras. 
El Hospital de Día se realiza los días miércoles y jueves, entre las 10 am. y la 1 pm., tiempo en el que se citaba de tres a cuatro adultos mayores. Lamentablemente, en muchas ocasiones, no venían todos a la consulta.

En la consulta de Neuropsicología el doctor Infantes realizaba una entrevista al adulto mayor y a su acompañante, que puede ser un familiar cercano o su cuidador. Durante la entrevista era tarea del practicante hacer apuntes sobre los temas más relevantes y sintetizar la información. En la entrevista se indaga acerca de si el paciente presenta olvidos, con qué frecuencia ocurren y de qué tipo son; también se hacen preguntas sobre el estado anímico, orientación en el espacio y tiempo al igual que la atención.

Luego de esto se da inicio a la aplicación del Test de Alteración de Memoria (T@M) que mide memoria inmediata, memoria de orientación temporal, memoria remota, memoria de evocación libre y memoria de evocación con pistas (Custodio et al., 2017). Se eligió esta prueba ya que la memoria que es la capacidad que tiene la persona para retener la información que uno adquiere (Valencia, Morante y Soto, 2014) y poder manipularla es un indicador sensible de la reducción de resto de funciones cognitivas (Leite, Castioni, Kirchner y Hildebrandt, 2015). El doctor Infantes y el practicante se dividían las evaluaciones, mientras uno evalúa el otro observa y toma apuntes. Además, en caso uno de los dos quisiera dar una apreciación o hacer una pregunta acerca de algo que no quedaba claro podía hacerlo sin ningún problema.

Una vez concluida la evaluación se despedía al paciente y se realizaba un pequeño resumen de un par de líneas donde se describen los hechos e información más relevante rescatada de las sesión y la calificación del test evaluado; todo esto era agregado a la historia clínica del adulto mayor. 
Al finalizar el día, todas las áreas del departamento de Geriatría se reúnen para compartir los resultados encontrados. El doctor Infantes y el practicante exponían los casos y cada uno hablaba sobre los pacientes que evaluó. De igual manera, cuando uno de los dos terminaba de hablar el otro solía dar una apreciación crítica sobre lo encontrado.

\subsection{Día del Adulto Mayor}

En el Hospital Central FAP el Día del Adulto Mayor es un evento que busca brindar un espacio de reconocimiento, gratitud y júbilo donde los adultos mayores puedan desarrollar distintas actividades, donde se sienten felices y puedan desenvolverse con sus pares. todo un acontecimiento.

Para esta actividad se unen las áreas de Neuropsicología y Psicología Geriátrica, conformando un equipo de dos psicólogos y tres internos, quienes se reunieron durante una semana fuera del horario de trabajo para preparar las actividades.

Así se decidió que el stand en la feria estaría conformado por diversos juegos y actividades que estimularan los procesos cognitivos de los adultos mayores, a fin de estimular la memoria, atención, motricidad, lenguaje, resolución de problemas y más. La primera pregunta que se hicieron fue como harían para que los adultos mayores no pudieran elegir el juego, porque esto podría sesgarlos a repetir el mismo juego una y otra vez, dejando de estimular algún proceso cognitivo. Para superar ello, diseñaron una ruleta, hecha de cartón y forrada con papel de colores de aproximadamente un metro de diámetro, que estaba dividida en nueve opciones distintas que el adulto mayor giraría para seleccionar el juego que realizaría.

- La primera actividad diseñada fue "Refranes": escribieron distintos refranes incompletos en hojas de papel con un plumón grueso para que les sea fácil de ver 
y los adultos mayores debían completarlos y explicar sus significado. Por ejemplo, “de tal palo, tal ...", a fin de estimular la memoria y la comprensión.

- En el siguiente juego le preguntaban al competidor su nombre y lo escribían en una hoja en blanco de manera vertical. Después, el participante debía escribir en la hoja, una pequeña historia con las letras de su nombre como un anagrama estimulando su creatividad y lenguaje.

- Luego, se diseñó una actividad donde se estimulaban los sentidos de los adultos mayores. Se vendaban sus ojos y se hacía que a través de sus sentidos reconozcan distintos objetos. Tocarían arroz dentro de un frasco, olerían una cebolla, tocarían algodón, probarían chocolate, etcétera.

- A fin de estimular la memoria el adulto mayor debía encontrar dos imágenes iguales mientras éstas estuvieran boca abajo y se les tomaba tiempo.

- Otra actividad fue hacer que los adultos mayores armen rompecabezas, con piezas de distintos tamaños que eran difíciles de armar.

- Se mostraron imágenes recargadas de objetos y los participantes debían encontrar alguna cosa en particular como: un animal en específico, una persona, una cama, etcétera, estimulando su atención y concentración.

- En una pizarra, se escribieron operaciones matemáticas que los adultos mayores debían responder mentalmente.

- Finalmente, se jugó bowling, para estimular su motricidad.

El Día del Adulto Mayor se desarrolló conforme a lo planificado y, según lo avizorado, la mayoría de los adultos mayores querían jugar solo un juego, bowling, pero al tener la ruleta se impidió que esto sucediera. Sin duda, fue una experiencia distinta y muy gratificante, verlos compartir y divertirse, estimulando sus procesos cognitivos y su motricidad. 


\section{CAPÍTULO III: RESULTADOS DE LA INTERVENCIÓN}

Durante el tiempo que duró la rotación del autor del presente informe en Neuropsicología, este adquirió muchos conocimientos nuevos acerca de evaluación y diagnóstico de adultos mayores; atendiendo a un gran número de pacientes y estando en contacto con una casuística muy variada.

Durante los cuatro meses que estuvo en Neuropsicología, consulta externa fue el nivel de atención más concurrido, con 288 consultas de acuerdo a las estadísticas del Hospital Central FAP, que representan un aproximado del número real de personas atendidas ya que, a veces, se atendía como un favor especial a una sexta persona en el día, o a veces se programaban dos pacientes a la misma hora y esto aparecía como solo uno.

En el Hospital de Día se atendió a 24 pacientes de acuerdo al registro que maneja el departamento de Geriatría ya que se veían de uno a cuatro casos por día. Además, en algunas ocasiones, los pacientes no terminaban todo el circuito de consultas con los especialistas por distintos motivos, pero igual se revisaban a la hora de las exposiciones de caso.

Estos dos niveles de atención, desde el ámbito de la Psicología, hacen una gran aporte, principalmente por la data que se genera de todas las evaluaciones. El doctor Infantes guarda todas las pruebas que aplica, y ha empezado a digitalizarlas por temas de espacio, lo que le permite contrastar en el tiempo la evolución del deterioro cognitivo de los adultos mayores. Así validó el Neuropsi, el Test de Aprendizaje Auditivo-Verbal de Rey y el Adas-Cog en la población FAP con la ayuda de sus internos a lo largo de los años, para así poder brindar un ayuda de calidad y con sustento estadístico a los adultos mayores. Cada vez que una prueba es aplicada, es una ayuda a la psicología en general ya que sus resultados están siendo almacenados y serán presentados en un futuro en una investigación longitudinal que el doctor está realizando. 
Por otro lado, el simple hecho que haya un departamento de Geriatría en el Hospital Central FAP es una gran ayuda para los adultos mayores, ya que ahí pueden ser atendidos con un costo mínimo, siempre y cuando sean personal de la FAP en actividad, retiro o familia directa. También atienden al público en general pero a un costo mayor.

El personal del departamento de Geriatría se preocupa genuinamente por los adultos mayores, por lo que se hacen diversas actividades para que ellos puedan tener un espacio de diversión y de esparcimiento con sus pares. El autor de la presente investigación solo participó del Día del Adulto Mayor, pero se celebran muchas fechas importantes realizándose diversas actividades para que los adultos mayores puedan pasar un momento agradable y de alegría. 


\section{CONCLUSIONES}

- El Hospital Central FAP brinda un servicio holístico a una gran número de adultos mayores que son tratados por distintos profesionales especializados en la tercera edad.

- El área de Neuropsicología no cuenta con el personal necesario para la atención en consulta externa, lo hace que los pacientes tengan que esperar meses por un diagnóstico.

- El Hospital de Día es un espacio muy útil y valorado por los adultos mayores, ya que agiliza la evaluación de pacientes en riesgo.

- El Hospital de Día no se da abasto para lograr atender a todos los pacientes considerados en riesgo.

- Las exposiciones de casos del departamento de Geriatría ayudan al consenso de los diagnóstico y de las necesidades más importantes de los pacientes.

- Las actividades de recreación que se realizan para los adultos mayores generan un ambiente cálido y de confraternidad entre los adultos mayores y el personal del hospital. 


\section{RECOMENDACIONES}

- Seria recomendable que el área de Neuropsicología se amplíe debido a que los pacientes tienen que esperar meses para poder recibir una cita.

- De igual manera, sería adecuado que el servicio del Hospital de Día sea todos los días, no solo miércoles y jueves, para así poder evaluar a más pacientes en riesgo.

- Debería haber mejor comunicación entre las enfermeras que agendan las citas y el área de Neuropsicología para comunicarles que en la primera sesión los pacientes deben de ir acompañados, y que la evaluación toma cuatro sesiones. 


\section{REFERENCIAS}

Chumpitaz, Y. y Moreno, C. (2016). Nivel de funcionalidad en actividades básicas e instrumentales de la vida diaria del adulto mayor. Revista Enfermeria Herediana, 9(1), 30-36. doi: 10.20453/renh.v9i1.2860.

Custodio, N., Lira, D., Herrera-Pérez, E., Montesinos, R., Castro-Suarez, S., Cuenca-Alfaro, J. y Valeriano-Lorenzo, L. (2017). Memory alteration test to detect amnestic mild cognitive impairment and early alzheimer's dementia in population with low educational level. Frontiers in Aging Neuroscience, 9(278). doi: 10.3389/fnagi.2017.00278.

Custodio, N., Lira, D., Herrera-Pérez, E., Nuñez del Prado, L., Parodi, J., Guevara-Silva, E., ..., Cortijo, P. (2014). The memory alteration test discriminates between cognitively healthy status, mild cognitive impairment and alzheimer's disease. Karger Publishers, 4(2), 314-321. doi: 10.1159/000365280.

Infantes, I. (2011). Perfiles neuropsicológicos de las demencias tipo Alzheimer, vascular y mixta en pacientes adultos mayores que acuden a un centro geriátrico y gerontológico: aspectos comparativos (Tesis de Maestría en Psicología Clínica). Universidad Peruana Cayetano Heredia, Lima.

Infantes, I. (2016). Relación entre la memoria auditiva y el rendimiento neuropsicológico en pacientes de la tercera edad atendidos en el centro geriátrico y gerontológico de la FAP (Tesis de Doctorado en Neurociencias). Universidad Nacional Mayor de San Marcos, Lima.

Instituto Nacional de Estadística e Informática (INEI). (2018). Situación de la población adulta mayor. Julio-Agosto-Septiembre 2018. Informe Técnico, $N^{\circ} 4$ - Diciembre 2018. 
Recuperado de https://www.inei.gob.pe/media/MenuRecursivo/boletines/informetecnico-situacion-de-la-poblacionadulta-mayor_dic2018.pdf.

Instituto Nacional de Estadística e Informática (INEI). (2015). En el Perú viven más de 3 millones de adultos mayores. Recuperado de https://www.inei.gob.pe/prensa/noticias/en-el-peru-viven-mas-de-3-millones-deadultos-mayores-8570/.

Leite, M., Castioni, D., Kirchner, R. y Hildebrandt, L. (2015). Capacidad funcional y nivel cognitivo de adultos mayores residentes en una comunidad en el sur de Brasil. Enfermería Global, 14(37). doi: dx.doi.org/10.6018/eglobal.14.1.196461.

National Institute of Aging. (2015). Alzheimer's disease \& related dementia. Recuperado de https://www.nia.nih.gov/health/what-alzheimers-disease.

Organización Mundial de la Salud. (2016). La salud mental y los adultos mayores. Recuperado de http://origin.who.int/mediacentre/factsheets/fs381/es/.

Valencia, J., Morante, P. y Soto, M. (2014). Velocidad de procesamiento y memoria de trabajo en adultos mayores: implicancias para el envejecimiento cognitivo normal y patológico. Revista de Psicología, 1(1), 13-25. Recuperado de http://ucsp.edu.pe/investigacion/psicologia/wp-content/uploads/2014/01/Revista-dePsicolog\%C3\%ADa-1er-art\%C3\%ADculo.pdf. 
APÉNDICES 


\section{APÉNDICE 1: TEST DE APRENDIZAJE AUDITIVO-VERBAL DE REY}

TEST DE MEMORIA AUDITIVA

Apellidos y Nombre

Edad:

Sexo:

Grado de Instrucción:

Ocupación:

Lateralidad:

Examinador: Fecha:

Quince palabras comunes que son leidas diez veces al examinado, siguiéncose una evocación a cada lectura final de la prueba se lee una historia que contiere to ias ias paiabras presentadas en la lista. Estas deben ser reconocidas por el sujeto. Se estudis el numero de pjiabras repeticias en cada evocación y se traza una curva de aprendizaje.

Se valora también el número de lecturas necesarias para aprender la lista completa y del número de paiabras evocadas correctamente.

\begin{tabular}{|c|c|c|c|c|c|c|c|c|c|c|}
\hline Palation / Ensomos & 1 & 11 & III & iv & $v$ & vi & viI & vil & $\mathbf{i x}$ & $x$ \\
\hline \multicolumn{11}{|l|}{1 rabanito } \\
\hline \multicolumn{11}{|l|}{2 Conejo } \\
\hline \multicolumn{11}{|l|}{3 Piala } \\
\hline \multicolumn{11}{|l|}{ 4. Lechuge } \\
\hline \multicolumn{11}{|l|}{ 5. Mono } \\
\hline \multicolumn{11}{|l|}{ 6. Esmeraida } \\
\hline \multicolumn{11}{|l|}{ 7. Brocoli } \\
\hline \multicolumn{11}{|l|}{ 8. Halcsn } \\
\hline \multicolumn{11}{|l|}{ 9. 2afiro } \\
\hline \multicolumn{11}{|l|}{ 10. Tomate } \\
\hline \multicolumn{11}{|l|}{ 11. Pantera } \\
\hline \multicolumn{11}{|l|}{ 12. Diamante } \\
\hline \multicolumn{11}{|l|}{13 Berengena } \\
\hline \multicolumn{11}{|l|}{ 14. Cerdo } \\
\hline \multicolumn{11}{|l|}{ 15. Gema } \\
\hline TOTAL & & & & & & & & & & \\
\hline
\end{tabular}

CURVA DE APRENOQNE

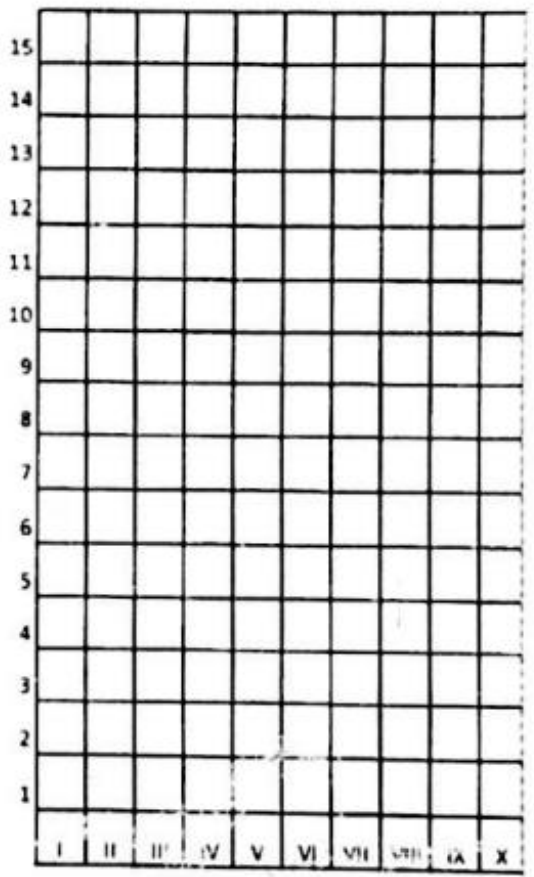

CONCLUSIONES: 


\title{
APÉNDICE 2: NEUROPSI
}

\section{Evaluación Neuropsicológica Brcve en Español NEUROPSI}

Apellidos y Nombres

Edad

Lugar de Nac

Escolaridad

Lateralidad

Motivo de Consuhta Derivado por

Observaciones de Importancia

\author{
HC \\ Procedencia \\ para una Evaluación Neuropsicologica
}

Ocupacion

Fecha de Evaluación.

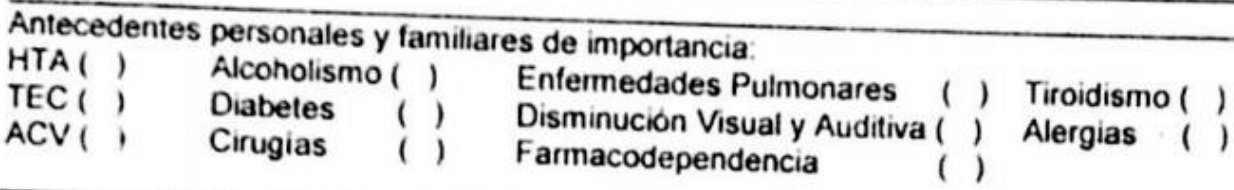

\section{ORIENTACION}

Tiempo : Dia ( ) Mes ( ) Año().

Espacio : Cludad () Lugar ().

Personal : Edad ().

PJE. TOTAL (6):

\section{ATENCIÓN Y CONCENTRACIÓN}

Digitos en regresión

4-8/9-1 (2)

Pje

8-6-3-2/2-6-1-7 (4

2-8-3/7-1-6 (3)

Detención Visual:

Aciertos:

6-3-5-9-1/3-8-1-6-2(5

Sustracción:

20/17/14/11/8/5

\section{Errores}

$\mathrm{Pje}$

Pje:

PJE. TOTAL (26):

\section{CODIFICACIÓN}

\begin{tabular}{l}
$\begin{array}{l}\text { A. Memoria Verbal Espontánea: Pje: } \\
\text { Intrusiones: } \\
\text { Primacia: }\end{array}$ \\
\begin{tabular}{|l|l|l|}
\hline \multicolumn{1}{|c|}{$\begin{array}{l}\text { Perseveraciones: } \\
\text { I Ensayo }\end{array}$} & II Ensayo & III Ensayo \\
\hline Gato & Mano & Codo \\
\hline Pera & Vaca & Fresa \\
\hline Mano & Fresa & Pera \\
\hline Fresa & Gato & Vaca \\
\hline Vaca & Codo & Gato \\
\hline Codo & Pera & Mano \\
\hline Total & Total & Total \\
\hline B. Proceso Visoespacial & Pje: \\
\hline
\end{tabular} \\
\hline
\end{tabular}

Fiuidez Fonologica: $\mathrm{N}^{\bullet}$ pal.

\section{LENGUAJE}

A. Denominación:

1. chivo_ 12. guitarra

Pje

4. dedo

15. llave

7. reloj 8 bicicleta

B. Repetición:

1. Sol_/2. Ventana _ /3. El niño llora 13. trompeta /6. serpiente

4. El hombre camina lentamente por la calle

C. Comprensión

1. Sefiale el cuadudo pequeno

2. Sefale un circuln y $: 1$ cuedrado

3. Sefale un circulo pequeno y un cuadrado grande

4. Toque el circulo pequeño si hay un cuadrado grande

5. Toque el cuadrado grande en lugar del circulo pequeno

6. Adernas de tocar los circulos. toque el cuadrado pequeno
D. Fluidez Verbal
Fluidez Semántica: $N^{\bullet}$ pal
Pje: $=\overline{\text { Pje. }}$ $=$ Pje 
Fluidez Somantica

$\begin{array}{ll}\text { Puntaje Natural } & \text { Valor Codificado } \\ 0-8 & 1 \\ 9-18 & 2 \\ 19-24 & 3 \\ 25-50 & 4\end{array}$

Fluidez Fonologica

Puntaje Natural Valor Codificado

0.6

7.13

14-18

$19-50$

V. LECTURA PJE. TOTAL (3):

1.- ¿Por qué se ahogó el gusano?

2.- ¿Qué pasó con el otro gusano?

3. ¿Cómo se salvó el gusano?

V. ESCRITURA PJE. TOTAL (2)

A) Dictado: El perro camina por la calle

B) Copiado: Las naranjas crecen en los árboles.

VII. FUNCIONES EJECUTIVAS
A) CONCEPTUAL:
Semelanxes:
1. Naranla/Pera
Pje Total
Pje(6)
2 Perro/Caballo
3 Ojo/Nariz
Cálculo:
$113+15=$
$P_{j e}(3)$
12 soles $+9-14=$
3 2\% docena $=$
Secuenciación
B) MOTORA:
Posición de la mano
Derecha
Izquierda
Movimientos altem
Reacciones Opuestas:

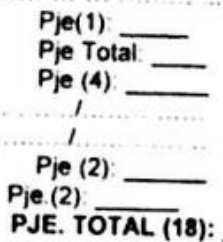

VIII. FUNCIONES DE EVOCACION

A. Memoria Visoespacial:

B. Memória Verbal:

Pje

\begin{tabular}{|c|c|}
\hline Evocación & C/clave \\
\hline & Partes del cuerpo \\
\hline & Frutas \\
\hline & \\
\hline & Animales \\
\hline $\operatorname{Pje}(6)$ & $\operatorname{Pie}(6):$ \\
\hline
\end{tabular}

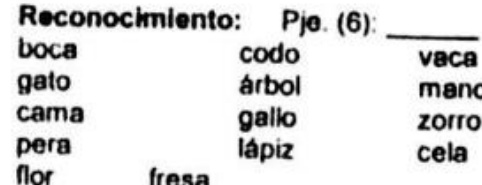

Intrusiones:__ Perseveraciones

Primacia:_Presencia

RESUMEN GENERAL

\begin{tabular}{|l|l|}
\hline \multicolumn{1}{|c|}{ Áreas } & Totales \\
\hline Orientación & \\
\hline Atención Concentración & \\
\hline Codificación & \\
\hline Lenguaje & \\
\hline Lectura & \\
\hline Escritura & \\
\hline Funciones Ejecutivas & \\
\hline Funciones De Evocación & \\
\hline
\end{tabular}

PUNTAJE GENERAL:

\begin{tabular}{|c|c|c|c|c|}
\hline \multicolumn{5}{|c|}{ Escolaridad Nula } \\
\hline Edad & Normal & Leve & Moderado & Sovero \\
\hline $16-30$ & $91-60$ & $69-46$ & $44-30$ & 29.14 \\
\hline $31-60$ & 84-68 & $67-64$ & $53-41$ & 40-28 \\
\hline $51-66$ & $90-69$ & $68-44$ & 43-28 & 27.13 \\
\hline $66-85$ & $76-48$ & 47.34 & $33-20$ & $19-8$ \\
\hline \multicolumn{5}{|c|}{ 1.4anos } \\
\hline Edad & Normal & Leve & Moderado & Severo \\
\hline $16-30$ & $104-73$ & $72-58$ & $67-42$ & $\mathbf{4 1 . 2 7}$ \\
\hline 31.50 & 104-81 & $80-69$ & $68-60$ & $57-46$ \\
\hline $51-65$ & 97.77 & $76-67$ & $66-67$ & $68-47$ \\
\hline $66-85$ & 8961 & $60-46$ & 48-32 & 31.18 \\
\hline
\end{tabular}

\begin{tabular}{|c|c|c|c|c|}
\hline Edad & Normal & Leve & Moderado & Severo \\
\hline $16-30$ & $113-102$ & $101-97$ & $96-86$ & $86-76$ \\
\hline $31-60$ & $117-106$ & $105-101$ & $100-90$ & $89-79$ \\
\hline $61-65$ & $110-98$ & $97-91$ & $90-79$ & $78-67$ \\
\hline $68-85$ & $90-80$ & $79-72$ & $71-66$ & $56-39$ \\
\hline
\end{tabular}

Edad Eecolaridad 10.24 años

\begin{tabular}{|c|c|c|c|c|}
\hline Edad & Normal & Leve & Moderado & Sovero \\
\hline $16-30$ & $114-103$ & $102-98$ & $97-67$ & 86.77 \\
\hline $31-60$ & $112-102$ & $101-97$ & $96-89$ & 87.78 \\
\hline $61-68$ & $101-03$ & $92-98$ & 87.80 & $79-72$ \\
\hline $66-88$ & 91.78 & 77.72 & 71.59 & $50-40$ \\
\hline
\end{tabular}

CONCLUSIÓN DIAGNOSSTICA:

RECOMENDACIONES: 


\title{
APÉNDICE 3: ADAS-COG
}

\author{
Anexo 3: Evaluación Cognitiva \\ ADASCOG
}

Apeilidos y Nombres

Edad:

Lugar de Nac

Escolaridad:

Lugar de Nac.

Lateralidad:

Dx. Neurologico:

Ocupacion

Dx. Neuropsicológico

RMN:

TAC

EEG:

Otros:

Motivo de Consulta: Derivado por

Observaciones de Importancia:

para una Evaluacion Neuropsicologica

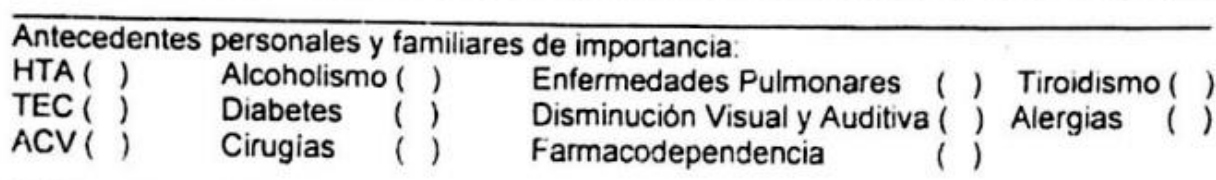

\begin{tabular}{|c|c|c|c|c|c|c|c|}
\hline \multicolumn{5}{|c|}{ 1. Inteligibilidad del lenguaje oral } & Tiempo & Señor & Señor \\
\hline 0 & 1 & 2 & 3 & 4 & Pugrta & Noche & Padre \\
\hline \multirow{2}{*}{\multicolumn{5}{|c|}{ 2. Comprensión }} & Orden & Cabeza & Puerta \\
\hline & & & & & Padre & Tiempo & Vaso \\
\hline 0 & 1 & 2 & 3 & 4 & Tiepra & Padre & Orden \\
\hline
\end{tabular}

3. F alta de palabra

3. F alta de palabra
\begin{tabular}{|c|c|c|c|c|c|}
\hline 0 & 1 & 2 & 3 & 4 & 5 \\
\hline
\end{tabular}

¿Cuál es su apellido? ¿De dónde es su familia? ¿Dónde nació? ¿Dónde estudió? ¿Se acueıda del nombre de alguno de sus companeros del colegio? ¿De quienes se acuerda? ¿Sabe algo de ellos? ¿Cużndo se caso? ¿En qué lugar? ¿Conocia a su esposa mucho tiempo antes de casarse? ¿Se acuerda de su primer trabajo? ¿Qué es lo que hacla? ¿A qué se debe que hoy en dia es más dificil para los jovenes obtener trabajo? ¿Tiene hijos? ¿Qué hacen? ¿Me puece nablar de eilos? ¿Qué piensa de la diferencia entre los jovenes y adultos mayores? ¿Donde vive? ¿Siempre ha vivido en ese lugar? ¿Le gusta el barrio donde vive? ¿Que hace durante el dia? ¿Mira TV? Muchas personas critican la calidad de los programas de $T V$ ¿ Qué es lo que piensa Ud de los programas de TV? ¿Tiene amigos? ¿Quienes son? ¿Los ve a menudo? ¿Tiene amigos comunes con alguno de sus amigos?

\section{Recuerdo de palabras}

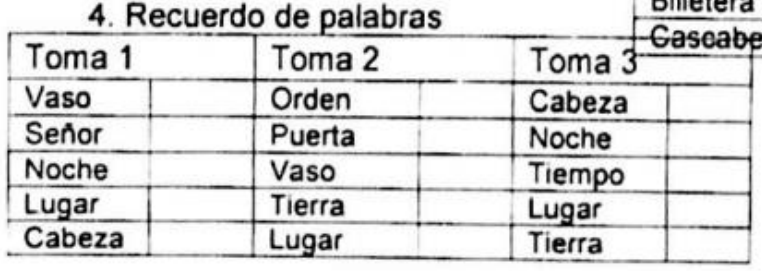

\begin{tabular}{|l|l|l|}
\hline \multicolumn{1}{|c|}{ Digitos } & Bien & Mal \\
\hline Pulgar & & \\
\hline Mayor & & \\
\hline Meñique & & \\
\hline Anular & & \\
\hline Indice & \\
\hline
\end{tabular}

\begin{tabular}{|l|l|l|}
\hline Objeto & $\checkmark$ & $X$ \\
\hline Rosa (crece en el lardin) & & \\
\hline Cama (se usa para dormir) & & \\
\hline Peineta (para el pelo) & & \\
\hline Lápiz (para escribir) & & \\
\hline Máscara (para disfrazar la cara) & & \\
\hline Tijera (para cortar papel) & & \\
\hline Lupa (para ver las cosas más grandes) & & \\
\hline Embudo (para llenar botellas) & & \\
\hline Flauta (instrumento de música) & & \\
\hline Pito (hace ruido cuando sopla) & & \\
\hline Billetera (para guardar billetes) & & \\
\hline Gaseabel (juguete de bebé) & \\
\hline
\end{tabular}




\section{Orientación}

\begin{tabular}{|l|l|l|}
\hline Preguntas & $\checkmark$ & $X$ \\
\hline ¿En qué dia de la semana estamos? & & \\
\hline ¿En qué ano estamos? & & \\
\hline ¿En qué estación estarnos? & & \\
\hline ¿En qué mes estamos? & & \\
\hline ¿Cual es la fecha de hoy? & & \\
\hline ¿Qué hora es? & & \\
\hline ¿En qué lugar estamos? & & \\
\hline
\end{tabular}

B. Dos rectángulos superpuestos

\section{Orientación de Ordenes}

\begin{tabular}{|l|l|l|}
\hline Orden & X X \\
\hline 1 Cierre el puño & & \\
\hline $\begin{array}{l}\text { 2 Muéstreme el piso y luego } \\
\text { el techo }\end{array}$ & & \\
\hline $\begin{array}{l}\text { 3. Ponga el lápiz encima del } \\
\text { sobre y vuélvalo a su lugar }\end{array}$ & & \\
\hline $\begin{array}{l}\text { 4. Ponga la peineta al otro } \\
\text { lado del lápiz y luego dé vuelta } \\
\text { al sobre }\end{array}$ & & \\
\hline $\begin{array}{l}5 . \text { Golpéese cada nombro dos } \\
\text { veces con dos dedos con los } \\
\text { ojos cerrados }\end{array}$ & \\
\hline
\end{tabular}

\section{Praxias}

"Yo quisiera que Ud. envie esta carta ponga el papel en el sobre, escriba nombre y su direcciton, ciérreic. péguelo y ponga la esta npilia"

\begin{tabular}{|l|l|l|}
\hline \multicolumn{1}{|c|}{ Instrucción } & Bien & Mal \\
\hline "Doble el papel" & & \\
\hline $\begin{array}{l}\text { "Meta la carta en el } \\
\text { sobre" }\end{array}$ & & \\
\hline "Pegar el sobre" & & \\
\hline "Escribir la & & \\
dirección" & & \\
\hline $\begin{array}{l}\text { "Pegar la } \\
\text { estampilla" }\end{array}$ & & \\
\hline
\end{tabular}

C. Rombo

Pje:

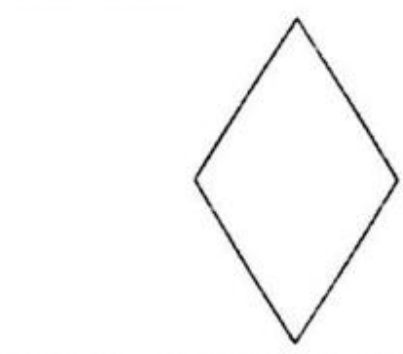

D. Cubo

Pje:

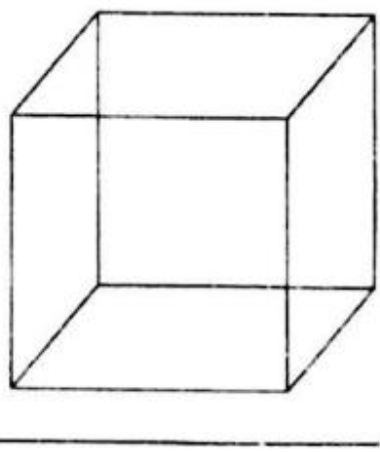

\section{Praxias Constructivas}

A. Circulo

Pje:
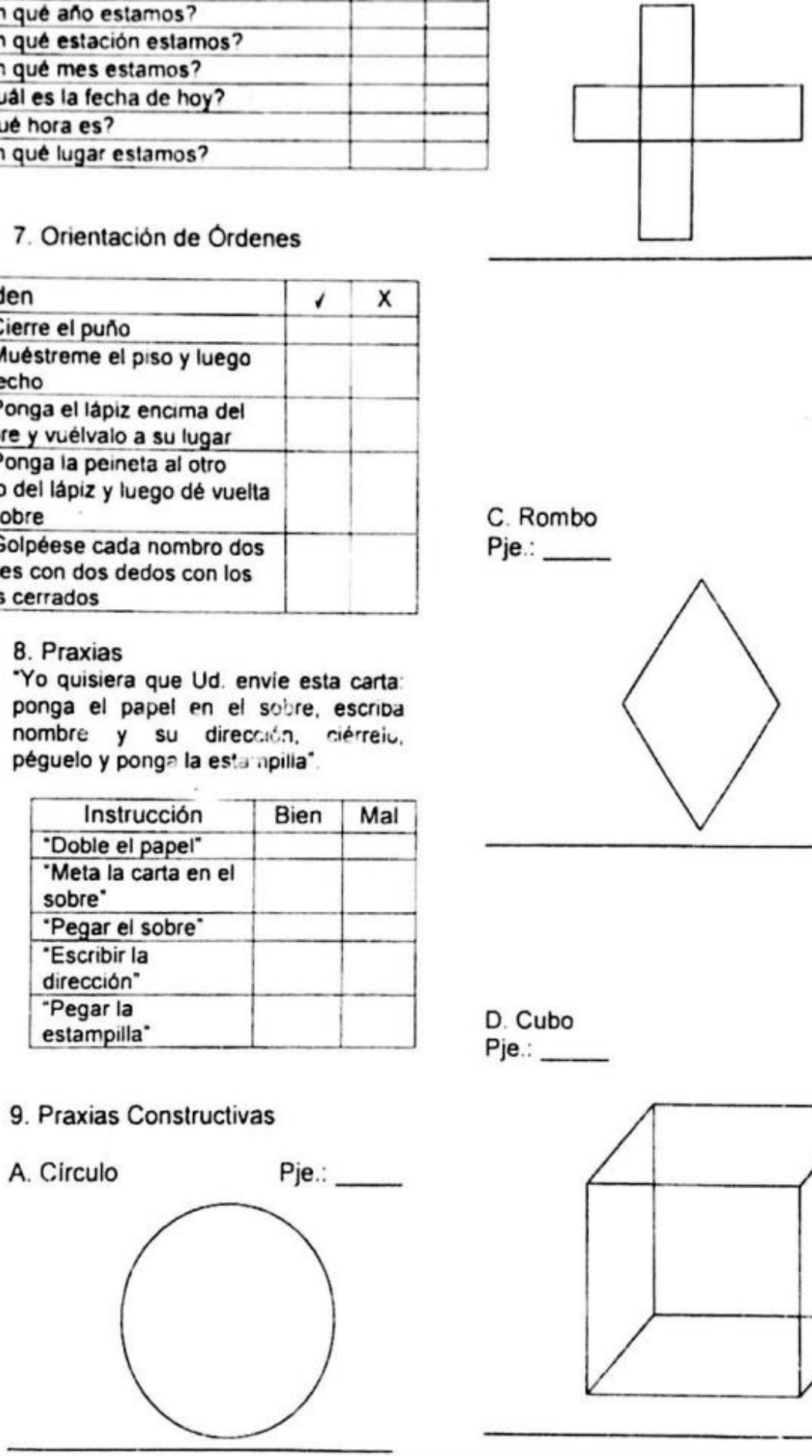
10. Reconocimiento de Palabras 1ra. Prueba

\begin{tabular}{|c|c|c|c|}
\hline \multirow{2}{*}{ Palabras } & \multicolumn{2}{|c|}{ Prueba 1} & \multirow{2}{*}{$\frac{\text { A } 1}{\text { Recuerdo }}$} \\
\hline & SI & $\mathrm{NO}$ & \\
\hline \multicolumn{4}{|l|}{ CIENCIA } \\
\hline \multicolumn{4}{|l|}{ Novela } \\
\hline \multicolumn{4}{|l|}{ Arte } \\
\hline \multicolumn{4}{|l|}{ AUTOR } \\
\hline \multicolumn{4}{|l|}{ Espera } \\
\hline \multicolumn{4}{|l|}{ RECIBO } \\
\hline \multicolumn{4}{|l|}{ Lectura } \\
\hline \multicolumn{4}{|l|}{ AMOR } \\
\hline \multicolumn{4}{|l|}{ Olvido } \\
\hline \multicolumn{4}{|l|}{ AFECTO } \\
\hline \multicolumn{4}{|l|}{ MADRE } \\
\hline \multicolumn{4}{|l|}{ Auto } \\
\hline \multicolumn{4}{|l|}{ Hospital } \\
\hline \multicolumn{4}{|l|}{ SIGLO } \\
\hline \multicolumn{4}{|l|}{ Gato } \\
\hline \multicolumn{4}{|l|}{ FAMILIA } \\
\hline \multicolumn{4}{|l|}{ CIUDAD } \\
\hline \multicolumn{4}{|l|}{ Pan } \\
\hline \multicolumn{4}{|l|}{ MUNDO } \\
\hline \multicolumn{4}{|l|}{ Peiola } \\
\hline \multicolumn{4}{|l|}{ PUEBLO } \\
\hline \multicolumn{4}{|l|}{ Perro } \\
\hline \multicolumn{4}{|l|}{$\mathrm{SO}$} \\
\hline PAGO & & & \\
\hline
\end{tabular}

Reconocimiento de Palabras: 2da Prueba

\begin{tabular}{l|l|l|l|}
\hline \multirow{2}{*}{ Palabras } & \multicolumn{2}{|c|}{ Prueba 2 } & A 1 \\
\cline { 2 - 3 } & Si & NO & Recuerdo \\
\hline Paso & & & \\
\hline AMOR & & & \\
\hline Ejemplo & & & \\
\hline PAGO & & & \\
\hline Entrada & & & \\
\hline Mente & & & \\
\hline MADRE & & & \\
\hline CREACION & & & \\
\hline Leche & & & \\
\hline PUEBLO & & & \\
\hline MUNDO & & & \\
\hline Militar & & & \\
\hline SIGLO & & & \\
\hline FAMILIA & & & \\
\hline Mar & & & \\
\hline CIENCIA & & & \\
\hline Edad & & & \\
\hline RECIBO & & & \\
\hline CIUDAD & & & \\
\hline Hambre & & & \\
\hline Café & & & \\
\hline AUTOR & & & \\
\hline LUna & & & \\
\hline AFECTO & & & \\
\hline
\end{tabular}

Reconocimiento de Palabras: 3ra. Prueba

\begin{tabular}{|l|l|l|l|}
\hline \multirow{2}{*}{ Palabras } & \multicolumn{2}{c|}{ Prueba 3 } & \multicolumn{1}{c|}{ A 1 } \\
\cline { 2 - 4 } & SI & NO & Recuerdo \\
\hline Lugar & & & \\
\hline AUTOR & & & \\
\hline Perdido & & & \\
\hline Mundo & & & \\
\hline Ciudad & & & \\
\hline Vuelta & & & \\
\hline CIENCIA & & & \\
\hline Vida & & & \\
\hline Siglo & & & \\
\hline FORMACION & & & \\
\hline RECIBO & & & \\
\hline Moneda & & & \\
\hline AMOR & & & \\
\hline ENFERMERA & & & \\
\hline Pago & & & \\
\hline GUERRA & & & \\
\hline Madre & & & \\
\hline BARCO & & & \\
\hline AFECTO & & & \\
\hline Mujer & & & \\
\hline Pueblo & & & \\
\hline MUUSICA & & \\
\hline Cielo & & \\
\hline FAMIILIA & & \\
\hline
\end{tabular}

11. Recuerdo de palabras

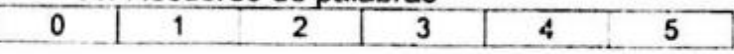

\section{RESUMEN GENERAL}

1. Inteligibilidad

2. Comprensión

3. Falta de palabra

4. Recuerdio de palabras

5. Denominación

6. Orientación

7. Orientación de Ordenes

8. Praxias

9. Praxias Constructivas

10. Reconocimiento de palabras

11. Recuerdo de palabras

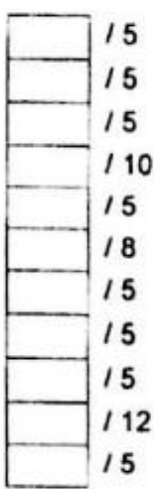


PUNTAJE TOTAL

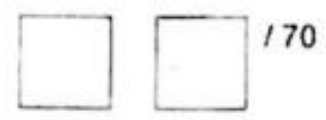

Puntaje Memoria

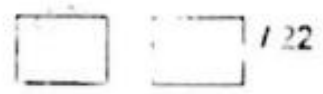

Recuerdo y reconocimiento de palabras

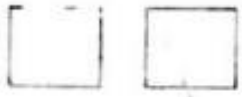

Puntaje Cognitivo 


\section{APÉNDICE 4: TEST DE ALTERACIÓN DE LA MEMORIA}

\section{TEST DE ALTERACION DE MEMORIA (TEM)}

MEMORLA INMEDLATA "Intente memorizar estas palabras. Es importante que esté atento/a" Repita: cerena (R) hacha (R) elefante (R) plano (R) verde (R)

1. Le he dicho una fruta, ¿cudl era?

2. Le he dicho una herramienta, ¿cusl era?

0 - 1 (SI 0, repetirla)

3. Le he dicho un animal, ¿cuall?

0-1

0-1

4. Le he dicho un instrumento musical, ¿cuśl?

5. Le he dicho un color, ¿cusl??

0-1

0-1

"Despues le pedin' que recuerde estos palabros"

"Este atenta/o a estas frases a intente memorizarlas" (míximo 2 intentos de repetición): Replta: TREINTA GATOS GRISES SE COMIERON TODOS LOS QUESOS (R)

6. ¿Cuántos gatos habla? 0-1; 7. ¿De qué color eran? 0-1; 8. ¿Qué se comieron? 0 - 1

(Si 0 decirle la respuesta correcta)

Replta: UN NIÑO UAMADO LUIS JUGABA CON SU BICICLETA (R) (máximo 2 intentos):

9. ¿Cómo se llamaba el niño? 0 - 1; 10. ¿Con qué juzaba? 0 - 1

(Si 0 decirle la respuesta correcta)

\section{MEMORIA DE ORIENTAQOON TEMPORAL}

11. Día semana 0-1; 12. Mes 0 - 1; 13. Día de mes 0 - 1; 14.Año 0 - 1; 15. Estación 0 - 1

MEMORIA REMOTA SEMANTICA (2 intentos; si error: repetir de nuevo la pregunta)

16. ¿Cuạ́l es su fecha de nacimiento?

$0-1$

17. ¿Cómo se llama el profesional que arregla autos?

0-1

18. ¿Cómo se llamaba el anterior presidente del gobierno?

$0-1$

19. ¿Cuál es el último día del año?

$0-1$

20. ¿Cuántos días tiene un año que no sea bisiesto?

$0-1$

21. ¿Cuántos gramos hay en un cuarto de kilo?

0-1

22. ¿Cuál es el octavo mes del año?

0-1

23. ¿Qué día se celebra la Navidad?

0-1

24. Si el reloj marca las 11 en punto, ¿en qué número se sitúa la aguja larga?

$0-1$

25. ¿Qué estación del año empieza en abril después del verano?

$0-1$

26. ¿Qué animal biblico engañó a Eva con una manzana? 0 - 1

27. ¿De que fruta se obtiene el pisco?

$0-1$

28. ¿A partir de que fruto se obtiene el chocolate?

0-1

29. ¿Cuánto es el triple de 1 ?

0-1

30. ¿Cuántas horas hay en dos dias?

0-1 


\section{MEMORMA DE BOCACON UREE}

31. De las palabras que dije al principlo, ¿cuales podria recordar? 0 - 1 - 2 - 3 - 4 - 5

(esperar la respuesta minimo 20 sezundos)

32. ¿Se acuerda de la frase de los catos? 0 - 1 - 2 - 3 (un punto por idea: 30 - erises - quesos)

33. ¿Se acuerda de la frase del ninho? 0-1-2 (un punto por idea: Luis - bicicleta)

\section{MEMORIA DE EVOCACON CON PISTAS}

34. Le dije una fruta, Lcuál era? 0-1

35. Le dije una herramienta, ¿cual? 0 -1

36. Le dije un animal Lcuśl era? 0 -1

37. Un instrumento musical, ¿cuál? $\quad 0-1$

38. Le dije un color, ¿cuál? $0-1$

¿Se acuerda de la frase de los gatos?

39. ¿Cuántos gatos habla? 0-1

40. ¿De que color eran? $0-1$

41. ¿Qué comían? 0-1

¿Se acuerda de la frase del niho?

42. ¿Cómo se llamaba? 0-1

43. ¿Con qué estaba jugando? 0-1

(Puntuar 1 en las ideas evocadas de forma libre)

\section{Normas de Puntuadín:}

Se suman todas las repuestas correctas (puntuación izual a 1). Se recomienda dar una puntuación global (sobre los 50 puntos miximos de la prueba) asi corno las puntuaciones pardales obtenidas por cada sujeto en cada uno de los apartados siguiendo el orden de valoraclón.

Ejemplo; TeM $=40(8,4,14,6,7)$

Indica que el sujeto ha obtenido una puntuación global de $\mathbf{4 0}$ sobre 50 , con una puntuación de 8 en el apartado de memoria inmediata, 4 en el de orientación, 14 en el de mernoria remota, 6 en el de evocación libre y 7 en el de evocackón con plstas. 